BULLETIN Bulletin hispanique

HISPANIQUE Université Michel de Montaigne Bordeaux

114-1 | 2012

Varia

Christine Pérès, Les jeux de la création et de la réception dans le roman mosaïque. Lecture de Sefarad d'Antonio Muñoz Molina

Peter Lang, Berne, 2011

Jean Alsina

(2) OpenEdition

Journals

Édition électronique

URL : http://journals.openedition.org/bulletinhispanique/1969

DOI : 10.4000/bulletinhispanique.1969

ISSN : $1775-3821$

Éditeur

Presses universitaires de Bordeaux

Édition imprimée

Date de publication : 1 juin 2012

Pagination : 492-495

ISBN : 978-2-86781-812-7

ISSN : 0007-4640

Référence électronique

Jean Alsina, "Christine Pérès, Les jeux de la création et de la réception dans le roman mosaïque. Lecture de Sefarad d'Antonio Muñoz Molina », Bulletin hispanique [En ligne], 114-1 | 2012, mis en ligne le 27 mai 2013, consulté le 22 septembre 2020. URL : http://journals.openedition.org/bulletinhispanique/1969 DOI : https://doi.org/10.4000/bulletinhispanique.1969

Ce document a été généré automatiquement le 22 septembre 2020

Tous droits réservés 


\section{Christine Pérès, Les jeux de la création et de la réception dans le roman mosaïque. Lecture de Sefarad d'Antonio Muñoz Molina}

Peter Lang, Berne, 2011

Jean Alsina

\section{RÉFÉRENCE}

Christine Pérès, Les jeux de la création et de la réception dans le roman mosaïque. Lecture de Sefarad d'Antonio Muñoz Molina. - Berne, Peter Lang, 2011, 437 p. - ISBN 978-3-0343-0322-4

1 Voici un livre important sur une œuvre majeure d'Antonio Muñoz Molina et in fine sur la question de l'écriture de l'Histoire. On sait qu'au-delà de la maîtrise d'une très belle écriture un des traits les plus attachants -et pour certains déroutants- de Sefarad (Alfaguara, 2001) est de rendre palpable (on osera dire vivable) la rencontre (l'irruption parfois), la constitution et l'assimilation progressive de la mémoire de l'épisode le plus tragique de notre histoire européenne récente par un sujet (un narrateur) espagnol contemporain que sa vie a priori banale ne prédispose pas à une telle aventure. Et qui souvent ne voit pas le lien avec sa propre histoire. L'entreprise repose sur le choix d'une construction fragmentée, proposée sans mode d'emploi ni lignes de suture entre ses parties qui peuvent se réclamer de genres littéraires différents; ce sont dix-sept récits qui « affichent ouvertement la tension entre la partie et le tout qui caractérise le texte» (p. 4 de couverture). Que lit-on? Un seul texte à trous? Un recueil de nouvelles? Que signifie «novela » dans le sous-titre «novela de novelas»? Comment lire la relation entre les deux tableaux (Nussbaum, Velázquez) qui ouvrent et ferment le texte? Quel rapport entretient ce parcours textuel avec l'histoire brûlante et l'héritage 
espagnol ? À ce propos une « Nota de lecturas » de trois pages donne en fin de volume les bases essentielles de la documentation de l'auteur et oriente la curiosité vers les frontières entre témoignage, histoire, mémoire et fiction. Christine Pérès a assimilé à son tour les informations et les questions portées par cette documentation qu'elle complète parfois et qui éclairent toute sa démarche. Mais elle va plus loin en considérant que la lecture de cette "novela de novelas" est elle-même une réelle aventure qui sollicite intensément l'activité du lecteur sans jamais dévoiler ouvertement ses règles et construit ce faisant l'éthique dont elle se réclame. Les polémiques médiocres auxquelles ce roman a pu donner lieu dès sa parution témoignent certes du malaise (explicable) ou de l'impéritie (peu excusable) de certains lecteurs. Mais d'autres lectures même attentives témoignent d'une perception incomplète des niveaux de jeu proposés, comme, par exemple, la non prise en compte du « recours à l'oralité et à l'image mentale » qui « efface le niveau énonciatif » dans le cas de « Berghof» (p. 215 et n. 36). En fait, c'est le cas de nombreux lecteurs et à suivre C. Pérès on a l'impression que la lecture peut être constamment approfondie et que la machine Sefarad s'avère inépuisable.

2 Cet ouvrage s'adresse donc de prime abord à tous les lecteurs comblés de Sefarad avides de mieux comprendre encore les soubassements et l'architecture de l'édifice qu'ils viennent d'habiter et souhaitent revisiter, tant l'apport d'information et l'analyse des parcours de lecture sont d'une grande richesse. Il peut aussi aider ceux qui se sont sentis perdus ou se disent restés sur leur faim tant la « relecture plus critique » qu'il construit avec succès «permet de mettre à jour un jeu d'amorces et de rappels passé inaperçu lors de la première lecture" (p.20). Plus largement il contribue à la compréhension et à l'illustration d'un type de roman si justement appelé "mosaïque » à la suite de l'étude de Lucien Dällenbach: Mosaïques (Paris, Seuil, 2001, citée, entre autres, p. 328 par C. Pérès). Dans la foulée, le chapitre final (II, 3) analyse la fonction du fragment, puis la "pensée fragmentaire ", pour déboucher sur une lecture de la trace, de la mémoire, de l'ironie, où Derrida est présent, vers une conception renouvelée de l'Histoire et du savoir. Il participe enfin avec succès au développement des travaux les plus actuels sur la réception du texte romanesque et sur les opérations de lecture. Le lecteur curieux trouvera dans quelques pages de l'introduction une très utile mise au point sur les voies et tendances des travaux sur la réception, suivies de la justification de la position de C. Pérès du côté de Milagros Ezquerro et de ses Fragments sur le texte (Paris, L'Harmattan, 2003) sans négliger un retour marqué vers Michel Picard et ses lectures : participative et distanciée. De cet ensemble de trois grandes voies d'analyse et de réflexion, où sont judicieusement mises à l'épreuve -entre autres- les diverses facettes des travaux de Vincent Jouve, se dégage une impression de cohérence honnête où la minutie du détail et la quête de vision d'ensemble font très bon ménage. On peut regretter que le pluriel du mot «jeu» choisi pour le titre puisse faire penser à une entreprise formaliste, au sens restreint du terme, alors que l'exploration de cet espace " où il y a du jeu » (p. 26) permet au contraire de déployer et de peser toute la gravité du sujet.

3 Le texte proprement dit comprend 406 pages. Il est suivi d'un utile «Repérage du découpage séquentiel de Sefarad " qui occupe dix pages et recense 151 séquences pour 17 titres. La bibliographie occupe 19 pages. Au passage on déplore l'absence d'un ou plusieurs index, notamment des œuvres et des passages cités, ainsi que des nécessaires titres courants. Puisque C. Pérès soutient à très juste titre (p.16) que Sefarad est « un 
véritable manuel d'apprentissage de l'acte de lecture" ou encore "d'une lecture critique des textes et de l'Histoire ", l'éditeur aurait pu avoir à cœur de fournir à son tour les outils d'un accès raisonné au texte critique forcément dense qui pourrait ainsi être abordé par plusieurs versants diversifiés. Un étudiant motivé lui en aurait su gré.

L'érudition, le souci constant du détail, la minutie de l'analyse, ne masquent pas (même si parfois le lecteur s'impatiente) l'aspect progressif de la démarche, son souci de globalité tournés vers la construction d'une véritable thèse. Un aperçu du sommaire en indique les grands traits. Une Première partie de 186 pages intitulée « Une esthétique du fragment " comprend deux chapitres: "De la transparence à l'illilisibilité : un péritexte ambigu» suivi de: "Un texte faussement transparent oscillant entre éclatement et unité ». La Deuxième partie (219 pages) intitulée : "Une poétique de l'inachèvement" se décline en trois chapitres dont le dernier synthétise une problématique englobante. On lit dans l'ordre : «Tension interne entre le playing et le game : les effets-personnage, entre jeux de rôle et jeux de l'esprit », "Tension externe entre le 'playing' et le 'game' : vers une unité diégétique » et «Une méditation sur la trace et le mal » suivi d'une brève conclusion qui conjoint, sur les pas de Hutcheon et de Ricœur, lecture du texte et lecture de l'histoire: «Porteur d'un discours utopique, Sefarad témoigne d'une volonté de faire entrer le récepteur dans le temps du possible, celui de l'uchronie, où l'humanisme pourrait encore vaincre l'individualisme et l'indifférence » (p. 406).

5 Parmi les axes de signification que le parcours de C. Pérès reconstruit ou, mieux, dévoile, on peut retenir d'abord l'analyse de la complexité d'une instance narrative multiple. Cette analyse permet de mettre en évidence la structure masquée de l'œuvre en montrant les deux niveaux mis en jeu, l'un qui met en scène un narrateur écrivain, l'autre qui conjoint les récits rapportés ou créés par lui. Sur cette base se construit dans la seconde partie une réflexion sur l'autofiction, sur l'auteur et ses masques qui prend en écharpe l'ensemble du texte. Un autre fil conducteur lui fait écho, celui qui met en place la présence d'un personnage récurrent, inaperçu au premier abord, récurrence qui éclaire à son tour le "retour en pointillé d'un narrateur écrivain » (G. Champeau) et donne accès à la structure du texte par la prise de conscience d'une architecture voilée. De là on peut faire retour à l'infinité des détails du texte, aux multiples recompositions, aux connexions et circuits de sens divers où cette étude entraîne son lecteur pris au jeu et promu acteur d'un permanent va-et-vient comme le présupposait le dispositif non déclaré de Sefarad. D'autant que la "première lecture", dite naïve et linéaire, que propose C. Pérès, en préalable à la « relecture critique », est textuellement argumentée, exempte de la subjectivité et des schémas psychologiques convenus qui décrédibilisent souvent ce type de tentative. On y adhère en général sans réticence, même si la notion de « lecteur réel » n'est pas toujours aisée à manier.

6 Une autre aire de jeu s'ouvre avec l'observation du découpage en récits et séquences. En dialogue avec les travaux d'Andrea Del Lungo, l'examen du statut et de la fonction de chacun des incipits et excipits et de leurs interrelations qui scandent la première lecture orchestre une " deuxième lecture critique " plus informée et mieux armée au cœur de «la capacité combinatoire multiple» des fragments (p. 334). L'analyse du départ du roman qui prend à contre-pied les attentes normées du lecteur y est pertinente et réussie. La partie consacrée à "Effet-personne et résonances sensorielles", dans I, 2 "Tension interne entre le playing et le game» (p. 195) en surprendra plus d'un. Elle met en place la construction d'une « mémoire charnelle » autour d'un lexique sensoriel 
qui prend en écharpe l'entier des récits et produit un effet puissant d'empathie, car, pour C. Pérès: "La vision du monde transmise par Sefarad est sous-tendue par un discours anthropologique, par une véritable anthropologie des sens, comme si, à l'instar des anthropologues, l'auteur avait conscience que le monde ne se donne à lire que sous forme de fragments, dans un feuilletage qui dit sa pluralité et sa complexité [...]» (p. 216). Autre piste encore celle que propose la réunion d'indices et d'analyses d'un « iconotexte » dont le livre dégage les axes et le fonctionnement qu'il s'agisse de l'art pictural, pour l'essentiel, du cinéma et de la photographie, directement ou indirectement présents; on ne peut qu'être convaincu, à la fin de II, 2, de ce que : "l'identité fondamentale [de Sefarad] se fonde précisément sur son rapport avec les arts plastiques» (p. 337). L'analyse de l'immense «Visión de España» de Sorolla est à cet égard on ne peut plus éclairante. On pourrait poursuivre par «la fusion de deux pratiques d'écriture, la nouvelle et la chronique " par exemple, mais je ne retiens ici bien arbitrairement que quelques aspects qui m'ont parus marquants.

7 La face la plus singulière et peut-être déroutante du texte est la constante tension qu'il installe, à l'instar de la mémoire et de l'Histoire, entre lisible et illisible. Ici aussi, le parcours que propose le livre de $C$. Pérès se révèle exhaustif et éclairant. Parler de l'illisible dans un texte si transparent (rien ici en effet de la complexité de la trame de Beatus ille) paraît une gageure. Brillament tenue par la recension exhaustive (souvent jubilatoire pour le lecteur) de la myriade de "leurres ", " chausse-trappes », " pièges " et autres "facteurs d'illisibilité de la structure romanesque»(p. 189). Intensément menée, la relecture les débusque avec bonheur tout au long des pages de Sefarad qui prennent ainsi l'allure d'une entreprise quasiment diabolique de destabilisation du lecteur, troublante et, au bout du compte, fraternelle (si on me permet ce terme), expérience aussi perturbante, me semble-t-il, que peut l'être le retour nécessaire sur notre histoire récente. C'est un des aspects les plus riches et originaux de l'ouvrage qui devient un éloge du trouble et de son dépassement, aux fondements d'une écriture de la mémoire fondée davantage sur l'éthique que sur le politique. C'est ici, à la fin de l'ouvrage, que peut apparaître le débat qui ne peut manquer de s'ouvrir entre ceux qui suivront C. Pérès, à travers sa grande connaissance de l'œuvre d'A. Muñoz Molina, sur les voies d'une lecture "ironique » de l'Histoire porteuse d'un « humanisme laïque » (p. 406) et ceux qui reprochent à Muñoz Molina une attitude révisionniste qui renvoie tout le monde dos à dos. C. Pérès montre bien l'ampleur de ce que Muñoz Molina rejette et combat et on ne peut refuser à son étude ni la rigueur ni la cohérence ni les heureuses références. C'est le mérite de cet ouvrage que d'unir dans une même coulée l'examen minutieux du détail au plus près de la matérialité du texte (ses " microanalyses ») et la vision surplombante qu'on peut en dégager sans jamais séparer ces deux versants. La quantité des faits textuels relevés est telle, leur ensemble si pertinent et convergent, si organisable en réseaux hiérarchisés que l'on en vient à se poser la question de la maitrise du projet par un auteur calculateur au point qu'on aimerait connaître la lecture que Muñoz Molina (qui a préfacé un précédent livre de C. Pérès) ferait de cette étude. On s'en tiendra à ce que dit $C$. Pérès des masques et de l'autofiction à propos du chapitre « Münzenberg » (p. 331) : « Le livre que le lecteur réel tient entre ses mains ressemble à celui que rêve d'écrire le personnage écrivain ».

8 Cet ouvrage prend pleinement place dans le paysage de l'hispanisme. Les très nombreuses références à des récits et romans contemporains, à des œuvres d'art, aux travaux d'historiens le relient aux pratiques d'écriture et de représentation contemporaines. Je suggérerais par exemple, à sa lumière, un retour sur Las semanas del 
jardín de Juan Goytisolo. Sur ses traces, la réflexion sur l'histoire pourra se prolonger avec Jorge Semprún (souvent cité) et peut-être José María Ridao. La lecture de $E l$ espectáculo de la creación y la recepción : Juegos de la edad tardía de Luis Landero de Elvire Gomez-Vidal Bernard (P.U. Bordeaux, 2010) permettra de comparer démarches et méthodes sur le terrain de la réception. Comme tout ouvrage académique celui-ci n'est pas exempt de lenteurs et de pointillisme démonstratif, de révérence devant ses sources. Cela permet à tout lecteur averti d'évaluer et de valider (ou non) en toute clarté les opérations de lecture, ce dont on lui sait particulièrement gré. Si ce travail se clôt sur la notion de « Texte-Acteur» (Florent Gaudez) il est aussi une belle promotion du lecteur actif et un bel hommage à un texte que tous n'osent affronter et qui est ici parfaitement balisé, alors même que son analyse donne le désir de poursuivre la partie. 